\title{
Polyamine Application Effects on Gibberellic Acid Content in Creeping Bentgrass during Drought Stress
}

\author{
Sanalkumar Krishnan and Emily B. Merewitz ${ }^{1}$ \\ Department of Plant, Soil, and Microbial Sciences, Michigan State University, 1066 Bogue Street, \\ East Lansing, MI 48824
}

\begin{abstract}
AdDitional INDEX wORDs. plant hormones, abiotic stress, turfgrass, water stress, Agrostis stolonifera
Abstract. Polyamines (PAs), spermine (Spm), and spermidine (Spd) may enhance the abiotic stress tolerance and growth of creeping bentgrass (Agrostis stolonifera). Growth chamber studies were conducted to investigate the effect of PA application on the physiological response and hormone content in creeping bentgrass 'Penn-G2' under drought. Spm (1 mM) and Spd (5 mM) were applied exogenously under drought or well-watered conditions. PA-treated plants maintained significantly higher turf quality (TQ), relative water content (RWC), photochemical efficiency, and membrane health while maintaining lower canopy temperature. Spm at the 1-mu rate had a 2.46-fold higher osmotic adjustment (OA) at 10 d compared with control plants. A greater content of gibberellic acid (GA) isoforms (GA1, GA4, and GA20) were observed compared with controls during both studies for PA-treated plants under drought. After 7 days of drought stress in Expt.1, GA1 levels were 3.26 higher for Spm 1-mm-treated plants compared with drought controls. GA4 contents were $69 \%$ and $65 \%$ higher compared with drought-stressed-untreated plants for Spd 5-mm application after 9 and 11 days. Higher levels of GA20 were observed at 10 days (Spd 5 mu, 108.9\% higher) due to PA treatment compared with drought controls. In addition to differential regulation of GA isoforms, we observed enhanced abscisic acid (ABA) due to PA application; however, not on a consistent basis. This study showed that PA application may play a role in GA1, GA4, and ABA accumulation in creeping bentgrass 'Penn G-2' under drought stress.
\end{abstract}

Polyamines are long-chain, aliphatic amines involved in the regulation of plant growth and response to environmental stresses including oxidative stress, drought, salinity, metal toxicity, and chilling stresses (Gill and Tuteja, 2010). PAs are also involved in basic processes such as protein synthesis and RNA and DNA processing (Cohen, 1998; JimenezBremont et al., 2014). The major PAs present in plants are putrescine [Put (diamine)], Spd (triamine), and Spm (tetramine) (Kusano et al., 2007). Specifically, for abiotic stress, PAs are known to be involved in stabilization of plant membranes and cellular structures by binding to membrane phospholipids. They also play a role in OA, modulating ion channels, act as reactive oxygen species scavengers, and can enhance antioxidant enzyme activity during stress (Pottosin and Shabala, 2014; Roychoudhury et al., 2011; Yamaguchi et al., 2007).

Exogenous application of Put, Spd, and Spm can be used to prime plants for stress tolerance. Gupta et al. (2012) reported that foliar spray of PAs in wheat (Triticum aestivum) significantly increased photosynthetic parameters, proline, total amino acids and soluble sugars, improved water status, grain yield, and reduced membrane damage under drought stress. Spd application to rice (Oryza sativa) significantly increased $\mathrm{K}^{+} / \mathrm{Na}^{+}$ ratio, $\mathrm{Ca}^{2+}$, and grain yield under salt stress (Saleethong et al., 2013). In a study with bermudagrass (Cynodon dactylon), Shi et al. (2013) reported that exogenous application of PAs (Spd, Spm, and Put) increased the activity of antioxidant enzymes

Received for publication 28 Nov. 2016. Accepted for publication 23 Feb. 2017. The authors thank the Michigan Turfgrass Foundation and AgBioResearch of Michigan State University for funding and support of this research.

${ }^{1}$ Corresponding author. E-mail: merewitz@msu.edu. and stress-related proteins under drought and salt stress. Because of their cationic nature, PA interacts with negatively charged macromolecules and stabilizes their structure during stress situations. The benefits of PAs application can be species specific and adequate rates of each PA for turfgrass species have yet to be determined; plants can exhibit phytotoxicity following PA applications. Therefore, determining whether PA applications may improve creeping bentgrass drought tolerance is warranted.

In addition to their role in stress tolerance, PAs are also known to regulate plant growth and development and are classified as plant hormones. PAs play a role in cell division and organ development (Davies, 1995). Recently, Shukla et al. (2015) showed that Spm and Spd applications promoted leaf growth and tillering rates in creeping bentgrass 'Penn G2' under optimal conditions, suggesting a role of PAs in regulating turfgrass shoots. Herein, we aim to determine whether creeping bentgrass shoot growth alterations by PAs may be associated with PA regulation of hormone accumulation in plant leaves.

Use of PAs as priming agents to better prepare plants for abiotic stress to improve turfgrass health and to promote growth has great potential practical application in the turf industry. Application of plant growth regulators is routinely employed in turf industry to regulate shoot growth, for seed head suppression and to improve turfgrass quality (Ervin and Koski, 2001). Therefore, the objectives of this study were to evaluate the effects of exogenous applications of Spd and $\mathrm{Spm}$ on growth physiology and accumulation patterns of phytohormones including GA isoforms (GA1, GA4, and GA20), salicylic acid (SA), jasmonic acid (JA), indole acetic acid (IAA), and ABA in creeping bentgrass under drought stress. We hypothesize that PAs may regulate GA isoforms to 


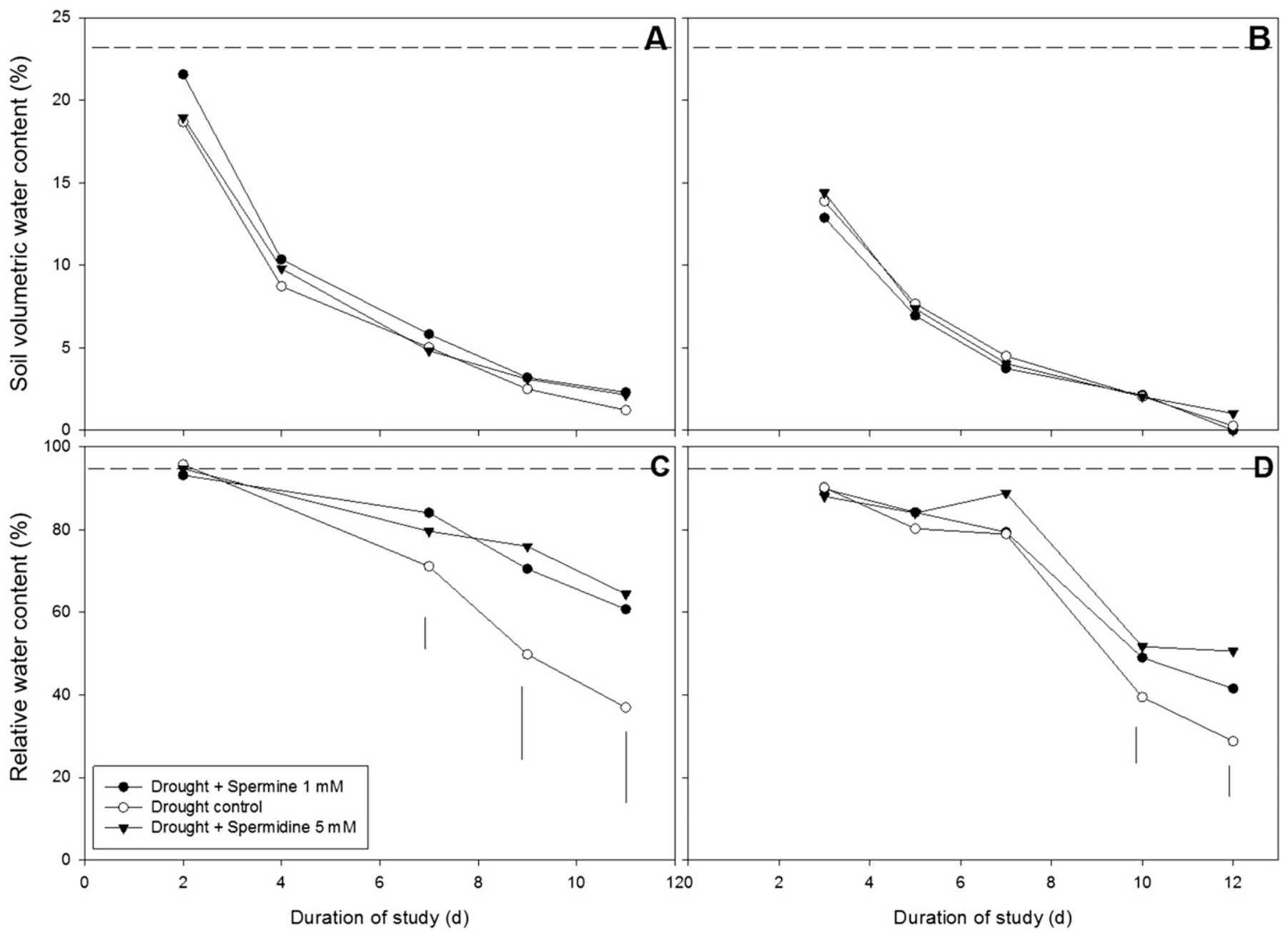

Fig. 1. Effect of polyamine (PA) application (spermine and spermidine) on soil volumetric water content of creeping bentgrass 'Penn-G2' under well-watered or drought conditions in (A) Expt. 1 and (B) Expt. 2. Leaf relative water content of creeping bentgrass leaves in response to water and PA treatment in $(\mathbf{C})$ Expt. 1 and (D) Expt. 2. A baseline mean value obtained from well-watered treatments is included. Least significant difference (LSD) bars are present on a given date where statistically significant differences were observed between chemical treatments $(P \leq 0.05)$. No LSD bar indicates no significant differences on that date. Treatment means were separated using Fisher's protected LSD $(n=4)$.

alter shoot growth and hormones involved in drought stress tolerance.

\section{Materials and Methods}

Plant material and growth Conditions. Creeping bentgrass 'Penn G2' sod pieces (10.16-cm diameter) were taken from Hancock Turfgrass Research Center in East Lansing, MI, on 18 July 2014 and established in 16 polyvinyl chloride pots (40 cm long, 10.5-cm diameter) filled with sandy loam soil ( $71 \%$ sand: $17 \%$ silt: $12 \%$ clay). Pots were covered at the bottom using a wired mesh to ensure adequate water drainage. The plants were irrigated using half strength Hoagland's solution and the soil moisture level was kept at full saturation. The chamber conditions included a light level of $560 \mathrm{mmol} \cdot \mathrm{m}^{-2} \cdot \mathrm{s}^{-1}$ ( $40 \mathrm{~cm}$ below the light source) with a $14-\mathrm{h}$ photoperiod, $21.5^{\circ} \mathrm{C}$ ambient temperature, and $65 \%$ relative humidity. Trimming was done every $3 \mathrm{~d}$ and plants were maintained at a $7.62-\mathrm{cm}$ height during the study period. Water was withheld from the drought-treated pots after the chemical treatment application. The study was repeated on 10 Oct. 2014 using 'Penn G2' plants.
Plants were grown in the same pots and under the same growth conditions as the first study.

PA treatments. Two growth chamber studies were conducted to evaluate the effect of Spm and Spd on creeping bentgrass plants. The treatments were applied on 18 Aug. 2014 as follows: 1) Spm at $1 \mathrm{~mm}, 2$ ) Spd at $5 \mathrm{~mm}$, and 3) control (water applied with no PA). Treated or control plants were then subjected to either well-watered or drought stress conditions (water completely withheld). All treatments were replicated four times. Thus, a total of 24 pots were used for each experiment. Spm and Spd were applied at the rate of $10 \mathrm{~mL}$ per pot using a handheld sprayer. The study was repeated to confirm the results from the previous study. Well-watered plants were irrigated daily until soil capacity was reached with $100 \mathrm{~mL}$ of water per pot.

Physiological evaluations. Soil volumetric water content (SVC) was measured using a soil moisture meter (TDR 100; Spectrum Technologies, Plainfield, IL) in a 0 - to 20-cm-deep soil layer of each pot by inserting the $20-\mathrm{cm}$-long rod vertically in the soil. TQ was a visual rating based on color, density, and uniformity of the grass canopy using a scale of $1-9[9=$ fully 


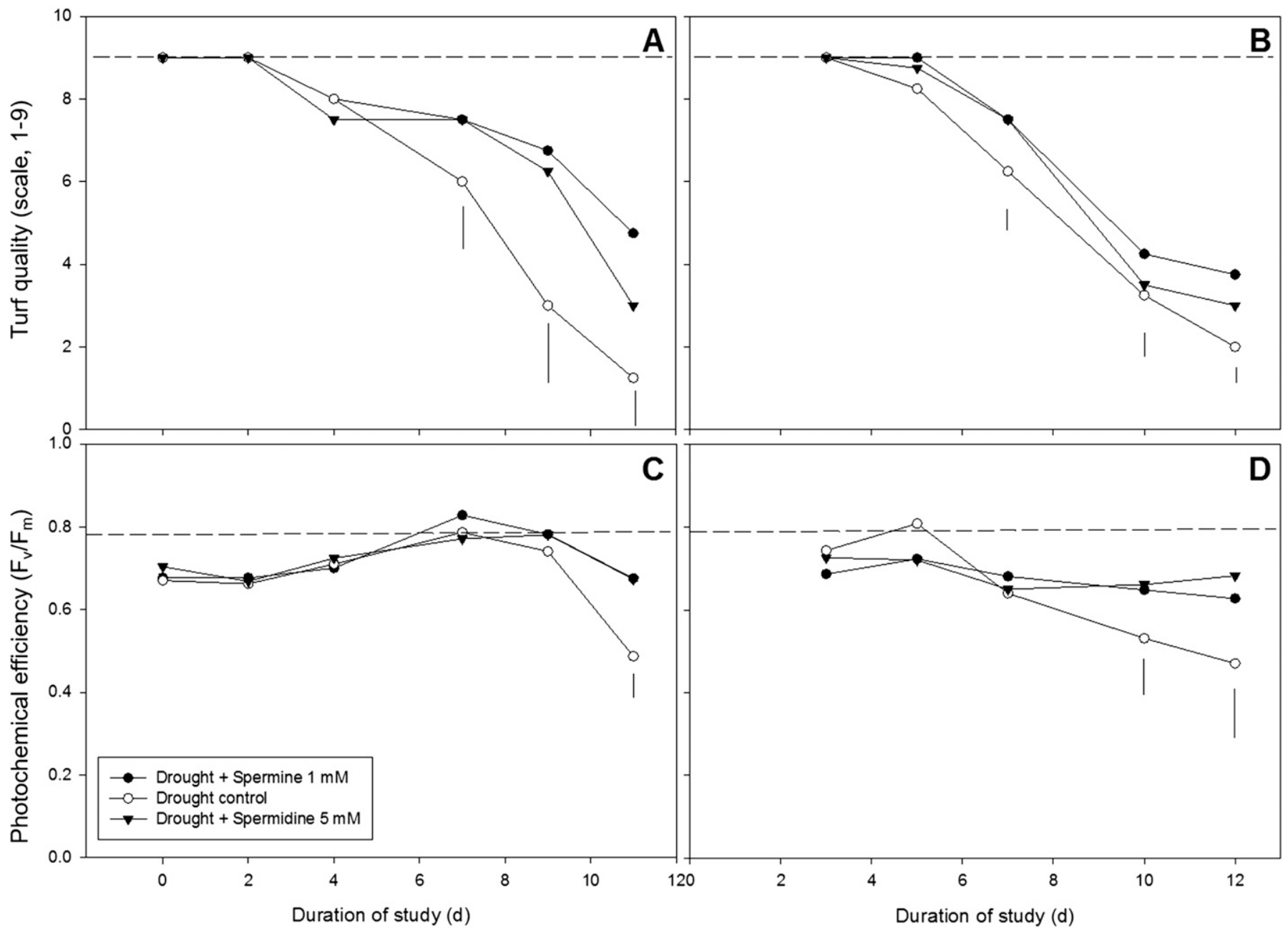

Fig. 2. Effect of polyamine (PA) application (spermine and spermidine) on turf quality ratings $(1=$ worst, $9=$ best) of creeping bentgrass 'Penn-G2' under wellwatered or drought conditions in (A) Expt. 1 and (B) Expt. 2. Photosynthetic efficiency $\left(\mathrm{F}_{\mathrm{V}} / \mathrm{F}_{\mathrm{M}}\right)$ of creeping bentgrass in response to water and PA treatment in (C) Expt. 1 and (D) Expt. 2. A baseline mean value obtained from well-watered treatments is included. Least significant difference (LSD) bars are present on a given date where statistically significant differences were observed between chemical treatments $(P \leq 0.05)$. No LSD bar indicates no significant differences on that date. Treatment means were separated using Fisher's protected LSD $(n=4)$.

turgid, dense green canopy, 1 = completely dead plants (Beard, 2001)].

Leaf RWC was determined using 10-12 fully expanded leaves per pot using the method described by Barrs and Weatherley (1962). About 10 mature leaves were harvested from plants and weighed to determine the fresh weight (FW). The leaves were placed in covered petri dishes filled with water and kept at $4{ }^{\circ} \mathrm{C}$ overnight to reach full hydration. Leaf samples were blotted dry and weighed to determine the turgid weight (TW). Leaf tissues were dried in an oven at $80{ }^{\circ} \mathrm{C}$ for $72 \mathrm{~h}$ and weighed to obtain the dry weight (DW). RWC was calculated as $(\mathrm{FW}-\mathrm{DW}) /(\mathrm{TW}-\mathrm{DW}) \times 100 . \mathrm{F}_{\mathrm{v}} / \mathrm{F}_{\mathrm{m}}$ and photochemical yield (YII) were determined with a flourometer system (OSp5; OptiSciences, Hudson, $\mathrm{NH}$ ) on intact leaves from plants with three subsamples or leaves per plant recorded.

Osmotic potential (MPa) was determined based on the osmotic potential of fully rehydrated leaves $\left(\psi_{\pi} 100\right)$ as described by Rachmilevitch et al. (2006). Harvested leaf tissues were fully hydrated using deionized water for $4 \mathrm{~h}$, blotted, and dried in tissue paper and immediately frozen in liquid nitrogen and stored at $-80^{\circ} \mathrm{C}$. At the time of measurement, leaf tissues were thawed at room temperature and cell sap was pressed and subsequently analyzed for osmolality [c (concentration of solution)], which measures the concentration of solution expressed as the total number if solute particles (millimoles) per kilogram in a vapor pressure osmometer (Vapro 5600; Wescor, Logan, UT). Osmolality of cell sap was converted to $\psi_{\mathrm{S}}$ in megapascals using the formula: $\Psi_{\mathrm{s}}=-\mathrm{c} \times 2.58 \times 10^{-3}$ (Krishnan and Merewitz, 2015). OA was calculated as the difference between osmotic potential of the drought-stressed leaves at full turgor from osmotic potential of nonstressed leaves at full turgor (Blum and Sullivan, 1986).

Phytohormone [zeatin riboside (ZR), SA, JA, ABA, IAA, GA1, GA4] extraction and quantification was based on the method from Liu et al. (2012) and modified as described for kentucky bluegrass (Poa pratensis) in the work of Krishnan and Merewitz (2014). About $200 \mathrm{mg}$ of frozen tissue sample was weighed and grounded to a fine powder in liquid nitrogen using mortar and pestle. Samples were weighed in $1.5-\mathrm{mL}$ tube, mixed with $850 \mu \mathrm{L}$ cold extraction buffer (methanol: water:acetic acid, 80:19:1, v/v/v), and vigorously shaken on a shaking bed for $16 \mathrm{~h}$ at $4{ }^{\circ} \mathrm{C}$ in the dark, and centrifuged at 


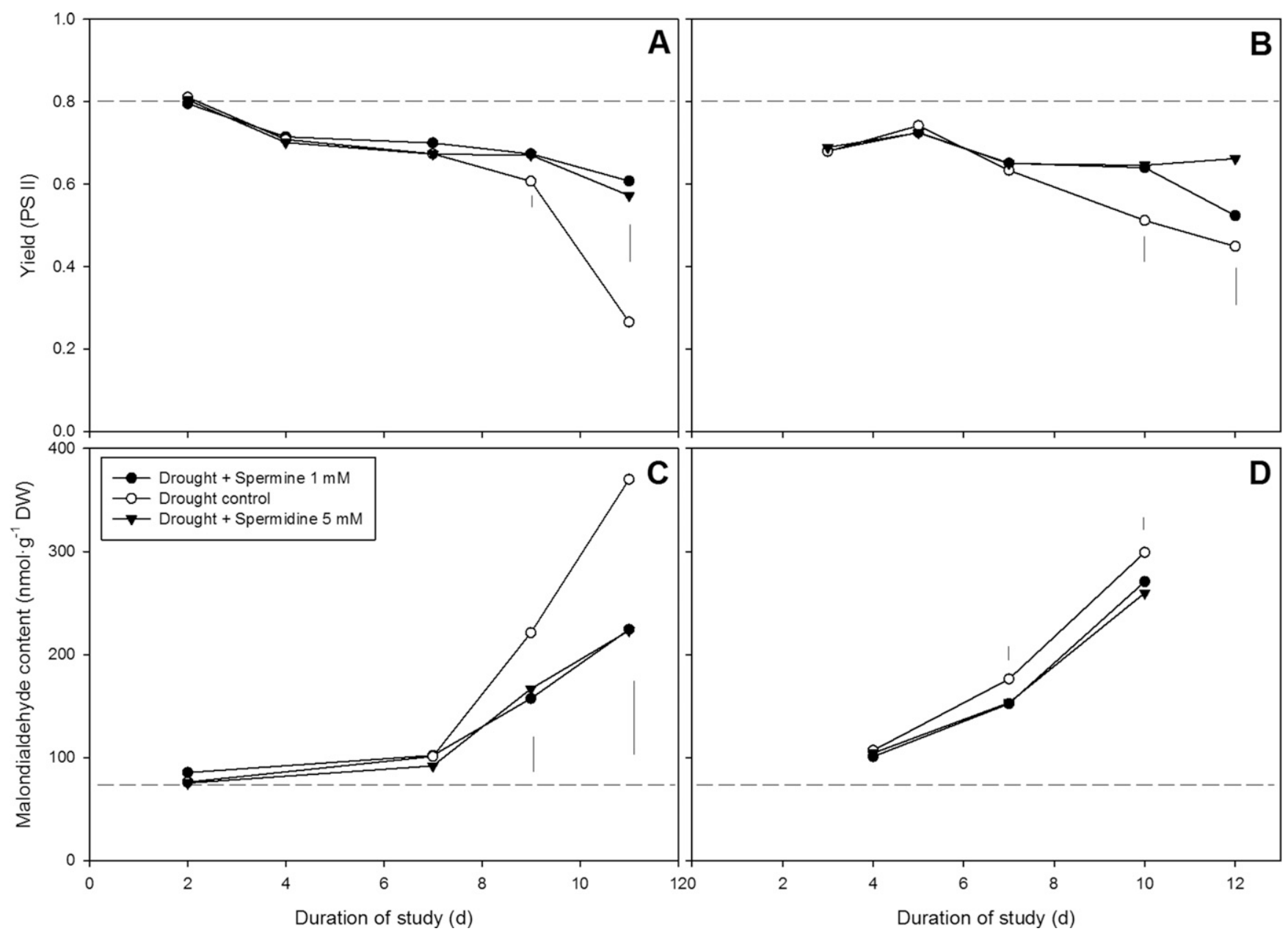

Fig. 3. Effect of polyamine (PA) application (spermine and spermidine) on photosynthetic yield (PSII) of creeping bentgrass 'Penn-G2' under well-watered or drought conditions in (A) Expt. 1 and (B) Expt. 2. Lipid peroxidation was measured based on malondialdehyde accumulation in creeping bentgrass in response to water and PA treatment in (C) Expt. 1 and (D) Expt. 2. A baseline mean value obtained from well-watered treatments is included. Least significant difference (LSD) bars are present on a given date where statistically significant differences were observed between chemical treatments $(P \leq 0.05)$. Treatment means were separated using Fisher's protected LSD $(n=4)$.

$10,976 g_{\mathrm{n}}$ for $20 \mathrm{~min}$ at $4{ }^{\circ} \mathrm{C}$. The supernatant was transferred to a new $1.5-\mathrm{mL}$ tube, and pellet was remixed with $400 \mu \mathrm{L}$ extraction buffer, shaken at $4{ }^{\circ} \mathrm{C}$ in the dark for $4 \mathrm{~h}$, and centrifuged at $10,976 g_{\mathrm{n}}$ for $20 \mathrm{~min}$ at $4{ }^{\circ} \mathrm{C}$. The supernatant from the two tubes was mixed and dried using centrifugal vacuum concentrator (Centrivap 78100-00; Labconco, KS City, MO) and then dissolved in $300 \mu \mathrm{L}$ methanol. The internal standard for liquid chromatography (LC) analysis included 100 nmol of deuterium-labeled ABA. LC was carried out using an ultra-high performance LC tandem mass spectrometer (Quattro Premier XE ACQUITY Tandem Quadrupole; Waters, Milford, MA).

Lipid peroxidation level was determined based on malondialdehyde (MDA) content using the method of Dhindsa et al. (1981) with modifications. A 1.0-mL enzyme solution was added to $2 \mathrm{~mL}$ of reaction solution containing $20 \%(\mathrm{v} / \mathrm{v})$ trichloroacetic acid and $0.5 \%(\mathrm{v} / \mathrm{v})$ thiobarbituric acid. The solution was heated in a water bath at $95{ }^{\circ} \mathrm{C}$ for $30 \mathrm{~min}$, cooled on ice, and centrifuged at $10,000 g_{\mathrm{n}}$ for $30 \mathrm{~min}$. The absorbance readings were taken at 532 and $600 \mathrm{~nm}$. The nonspecific absorbance at $600 \mathrm{~nm}$ was subtracted from absorbance at
$532 \mathrm{~nm}$ and MDA content was calculated using the adjusted absorbance and extinction coefficient of $155 \mathrm{~mm}^{-1} \cdot \mathrm{cm}^{-1}$ (Heath and Packer, 1968).

Statistical analysis and eXPERIMENTAL Design. Analysis of variance was based on the general linear model procedure of SAS (version 9.1; SAS Institute, Cary, NC). The pots were arranged in a completely randomized block design with four replications. The Fisher's protected least significant difference (LSD) test at the $0.05 P$ level was used to detect the difference between treatment means. LSD bars were presented in the figures where a statistically significant treatment effect was observed.

\section{Results}

SVC did not differ significantly within the well-wateredtreated plants for both experiments. Drought stress treatment caused a significant reduction in SVC in both experiments. PA treatments did not cause significant differences in SVC (Fig. 1A and B). Similarly, the well-watered plants did not show any significant difference in leaf RWC. PA-treated plants 




Fig. 4. Effect of polyamine (PA) application (spermine and spermidine) on osmotic adjustment (MPa) of creeping bentgrass 'Penn-G2' under well-watered or drought conditions at 3 and $10 \mathrm{~d}$. The data presented here are from Expt. 2. The results from drought-stressed and well-watered plants with PA application along with controls (no PA application) are presented here. Bars with different letters indicate significant difference for the treatments. Bars that share similar letters are not significantly different for the treatments $(P \leq 0.05)$. Treatment means were separated using Fishers protected least significant difference $(n=4)$.

maintained significantly higher RWC compared with droughtstressed controls for both experiments (Fig. 1C and D). During the first study, Spd 5-mu-treated bentgrass maintained $11.2 \%$, $52.7 \%$, and $74 \%$ higher RWC, whereas Spm 1-mu-treated plants showed $18 \%, 41.6 \%$, and $64.9 \%$ higher RWC at 7,9 , and $11 \mathrm{~d}$, respectively, compared with controls. In Expt. 2, Spd 5-mM- and Spm 1-mm-treated plants during drought had 26.3\%, $30.9 \%$, and $24.1 \%$ higher RWC than controls, respectively, at 10 d. At 12 d, Spd 5-mm- and Spm 1-mu-treated plants during drought maintained $60.2 \%, 75.9 \%$, and $44.2 \%$ higher RWC compared with controls.

Drought stress treatment caused a significant decline in TQ. TQ for PA-treated plants at 7, 9, and $11 \mathrm{~d}$ was significantly greater than drought-stressed controls during Expt. 1 (Fig. 2A). In Expt. 2, higher TQ was observed for Spm $1 \mathrm{~mm}$ at 7, 10 and $12 \mathrm{~d}$, respectively, whereas Spd $5 \mathrm{~mm}$ showed better quality at 7 and $12 \mathrm{~d}$ compared with untreated plants under drought (Fig. 2B). $F_{v} / F_{m}$ was higher for PA-treated plants during both experiments (Fig. 2C and D). Treatment with Spd (5 mM) and Spm (1 $\mathrm{mm})$ had $37.5 \%$ and $37.5 \%$ higher $F_{v} / F_{m}$ values, respectively, compared with control plants at $11 \mathrm{~d}$ during the first experiment. During Expt. 2, both Spm- and Spd-treated plants showed 30\% and 32\% higher $\mathrm{F}_{\mathrm{v}} / \mathrm{F}_{\mathrm{m}}$ than control plants at 10 and $12 \mathrm{~d}$ after treatment (DAT), respectively. YII was $11.6 \%$ higher for Spd 50-mm- and Spm 1-mM-treated plants at 9 DAT than controls during Expt. 1 (Fig. $3 \mathrm{~A})$. At $11 \mathrm{~d}$, the YII values were $11.9 \%$ and $130.7 \%$ higher for Spd 5-mм and Spm 1-mm treatments, respectively, compared with controls. In Expt. 2, both Spm- and Spd-treated plants had $25 \%$ higher YII than drought controls at $10 \mathrm{~d}$. At $12 \mathrm{~d}$, plants treated with Spd had significantly better YII than droughtstressed control plants (Fig. 3B).
Lipid peroxidation was lower on some days in PA-treated droughtstressed plants compared with control drought-stressed plants. For example, Spd-treated plants showed $24.6 \%$ and Spm-treated plants showed 28\% lower MDA content compared with controls during Expt. 1 (Fig. 3C). In Expt. 2, PAtreated plants (both Spd $5 \mathrm{~mm}$ and Spm $1 \mathrm{~mm}$ ) had 13\% lower MDA content at $7 \mathrm{~d}$ compared with drought control (Fig. 3D). At $10 \mathrm{~d}$, Spd- (5 mM) and Spm- (1 mM) treated plants had $13 \%$ and $19 \%$ lower MDA content during drought compared with drought-stressed controls, respectively.

OA was higher for PA-treated plants under severe drought stress compared with well-watered plants (Fig. 4). Spm 1-mm-treated plants had 2.46-fold higher OA at $10 \mathrm{~d}$ compared with control plants under drought stress.

We have measured the content of JA, SA, IAA, ZR, and GA20 in this study, but the data are not presented here due to lack of significant differences among chemical treatments (data not shown). Significant chemical treatment effects were detected for GA1, GA4, and ABA. The content of GA1 and GA4 for both Spd- and Spm-treated plants was greater than in control plants under drought stress during Expt. 1 (Fig. 5). After $7 \mathrm{~d}$ of drought stress, GA1 levels were 3.26 times higher for Spm 1-mM-treated plants compared with controls. GA4 levels were $69 \%$ and $65 \%$ higher for Spd 5-mm-treated plants after 9 and $11 \mathrm{~d}$, respectively. During Expt. 2, significantly higher GA1 levels were observed after $4 \mathrm{~d}$ (Spm $1 \mathrm{~mm}, 150 \%$ higher) and $10 \mathrm{~d}$ (Spm $1 \mathrm{~mm}, 104 \%$; Spd $5 \mathrm{~mm}, 106 \%$ higher) for the treated plants compared with controls (Fig. 5B). GA4 content was upregulated at $4 \mathrm{~d}$ (Spm $1 \mathrm{~mm}$, 74.4\% higher) and $10 \mathrm{~d}$ (Spm $1 \mathrm{~mm}, 234 \%$; Spd $5 \mathrm{~mm}, 63 \%$ higher) compared with controls (Fig. 5C). During Expt. 1, ABA content was 35\% higher in Spd 5-mm-treated plants after $11 \mathrm{~d}$ of drought stress (Fig. 6A). In Expt. 2, Spm 1-mm-treated plants showed higher upregulation of ABA (73\%) at $10 \mathrm{~d}$ (Fig. 6B).

\section{Discussion}

PAs played a protective role for enhanced survival of creeping bentgrass to drought stress via effects on photochemical health, membrane stability, and OA, which are all important drought tolerance mechanisms. PAs have been reported to be involved in the synthesis of other metabolites, such as proline, and can function as osmolytes to decrease water loss from cells and lead to maintenance of higher leaf water content in plants under stress conditions (Kotakis et al., 2014). The synthesis of osmoprotectants or compatible solutes is an important mechanism through which plants acclimate to water deficit conditions (Bartels and Sunkar, 2005). PA treatments significantly improved the photochemical health of creeping 

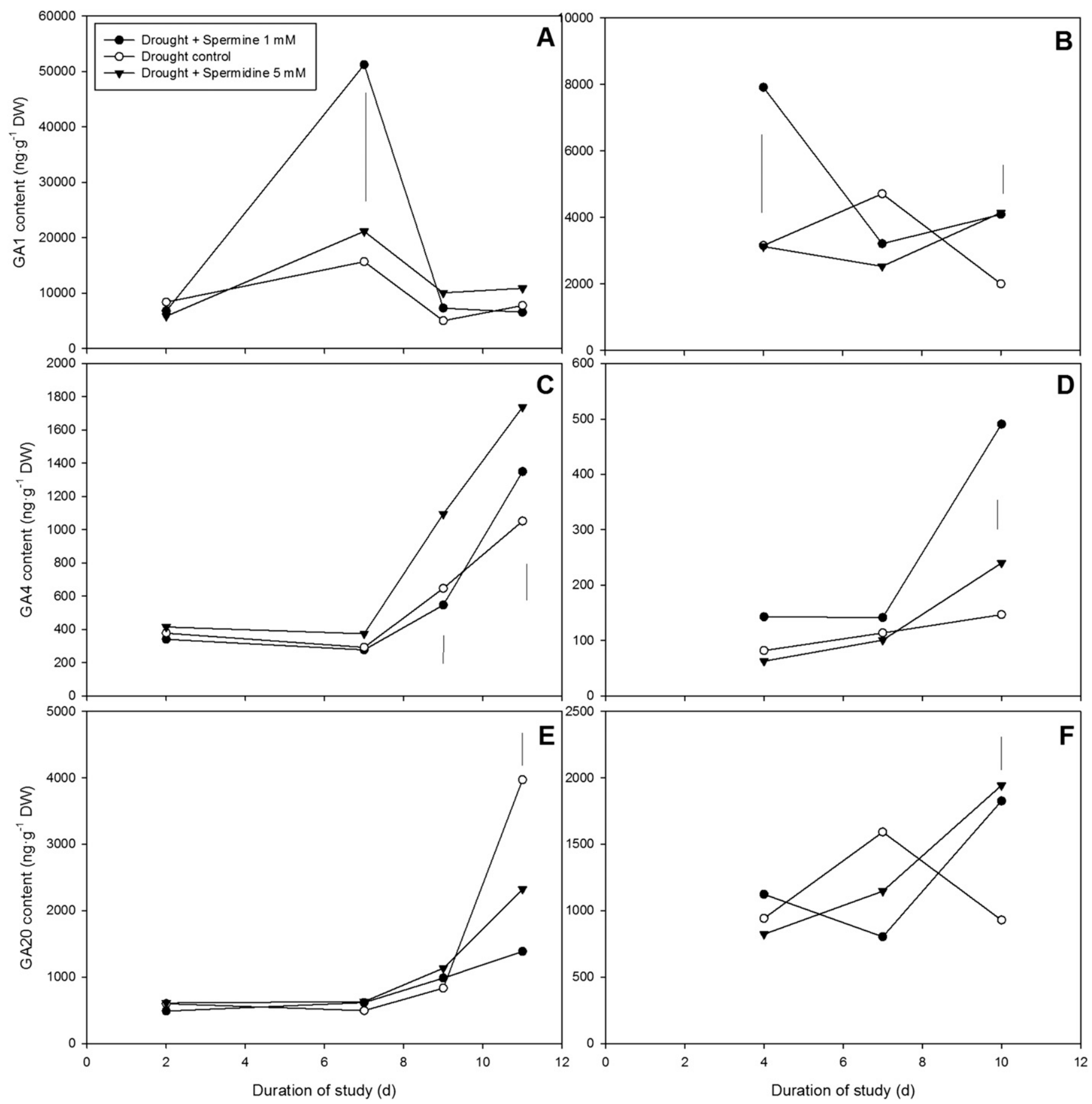

Fig. 5. Endogenous leaf hormone content of gibberellic acid (GA) isoforms: (A) GA1 in Expt. 1, (B) GA1 in Expt. 2, (C) GA4 in Expt. 1, (D) GA4 in Expt. 2, (E) GA20 in Expt. 1, and (F) GA20 in Expt. 2 in creeping bentgrass 'Penn G2' plants exposed to drought and polyamine (spermine and spermidine) application. Different letters indicate statistical significance within a given day of treatment. Letters are based on least significant difference values determined by Fisher's protected $t$ test $(P \leq 0.05)$.

bentgrass under drought, which was evident by the higher $\mathrm{F}_{\mathrm{v}} / \mathrm{F}_{\mathrm{m}}$ and YII observed during both experiments. The effects of PA on $\mathrm{Fv} / \mathrm{Fm}$ during drought are consistent with previous research (Shukla et al., 2015). The maintenance of cell membrane integrity and stability under water stress conditions is a major component of drought tolerance in plants. PA-treated plants showed lower electrolyte leakage and lipid peroxidation under water stress compared with control plants. Less lipid peroxidation is commonly associated with healthy antioxidant enzyme systems. Exogenous application of PA enhanced antioxidant enzymes in creeping bentgrass and those plants had greater membrane health and lower peroxidation compared with control plants (Shi et al., 2013).

PAs are a part of a complex signaling system that regulates stress tolerance, which gives them hormone-like function (Davies, 1995). The interactions of PAs and other hormones have not yet been fully elucidated. GA1 and GA4 are the most active isoforms of GA observed in plants, whereas GA20 is 

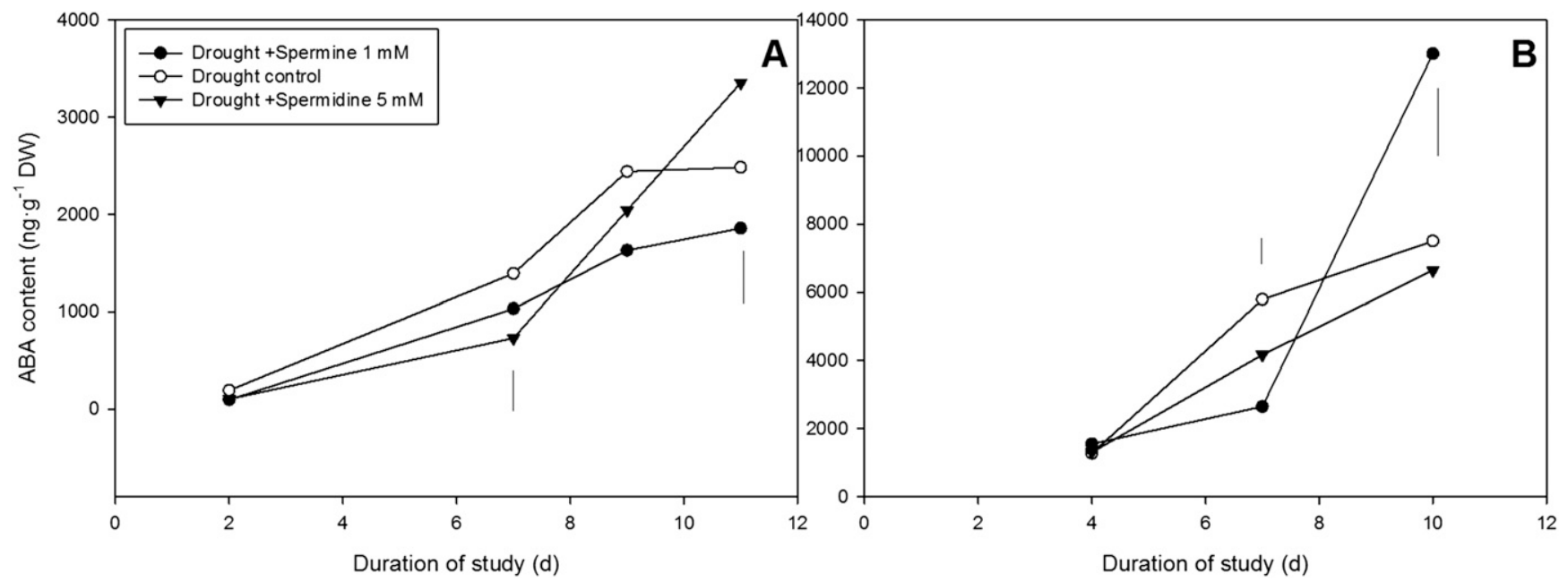

Fig. 6. Endogenous leaf hormone content of abscisic acid in (A) Expt. 1 and (B) Expt. 2 in creeping bentgrass 'Penn G2' plants exposed to drought and polyamine (spermine and spermidine) application. Different letters indicate statistical significance within a given day of treatment. Letters are based on least significant difference values determined by Fisher's protected $t$ test $(P \leq 0.05)$.

bioinactive but is an important precursor of GA1 and GA4. Bioactive GAs control plant growth and development, seed germination, stem elongation, leaf expansion, and flower and seed development (Yamaguchi, 2008). We found significantly higher GA1 and GA4 in Spm- and Spd-treated plants in both studies compared with controls, which suggests that both isoforms are regulated by PA treatment. Arginine decarboxylase (ADC), the key enzyme involved in the biosynthesis of PAs (Put, Spd, and Spm), was increased by the application GA3 to pea plants (Pisum sativum). The endogenous content of Put, Spd, and Spm were significantly increased due to the action of ADC, supporting the notion that PAs are regulators of GA (Dai et al., 1982). PAs may play a role in cell division in association with GA and PA levels may be increased by the application of GA (Kyriakidis, 1983; Shiozaki et al., 1998). Herein, we have found that application of PAs enhances GA content.

Drought stress increased ABA accumulation for both untreated and PA-treated plants. Higher content of ABA is often associated with greater drought tolerance in turfgrasses and could be responsible for the regulation of many stressprotective genes ( $\mathrm{Lu}$ et al., 2009). Our study showed that ABA content was significantly enhanced in Spd 5-mm-treated plants at $11 \mathrm{~d}$ during the first experiment and in the second study, ABA levels were higher than in control plants for Spm 1mM-treated plants at $10 \mathrm{~d}$. The current study shows that ABA may be affected by PA application; however, this was not consistent for all days or treatments. Alcazar et al. (2006) found increased expression of three PA biosynthesis genes ADC2, SPDS1, and SPMS under drought stress in Arabidopsis thaliana mutants impaired in ABA biosynthesis suggesting that PA could be involved in ABA regulation under drought stress. Further work would be required to elucidate the effects of PAs on ABA levels in creeping bentgrass under stress.

The results indicate that foliar application of PAs, Spd, and Spm may have a protective effect on creeping bentgrass under drought stress and this protection could be associated with hormone signaling. Higher levels of GA isoforms (GA1 and GA4) were detected during drought stress in response to PA application before drought stress. This suggests a role of PAs in regulating GA metabolism in creeping bentgrass and could be exploited for plant growth regulation or plant growth promotion purposes in the turfgrass industry. Further studies on the effect of PA application in turfgrass performance under field conditions are required for fully realizing its potential use as growth regulator.

\section{Literature Cited}

Alcazar, R., J.C. Cuevas, M. Patron, T. Altabella, and A.F. Tiburcio. 2006. Abscisic acid modulates polyamine metabolism under water stress in Arabidopsis thaliana. Physiol. Plant. 128:448-455.

Barrs, H.D. and P.E. Weatherley. 1962. A re-examination of the relative turgidity techniques for estimating water deficits in leaves. Austral. J. Biol. Sci. 15:413-428.

Bartels, D. and R. Sunkar. 2005. Drought and salt tolerance in plants. Crit. Rev. Plant Sci. 24:23-58.

Beard, J.B. 2001. Turf management for golf courses. 2nd ed. Ann Arbor Press, Chelsea, MI.

Blum, A. and C.Y. Sullivan. 1986. The comparative drought resistance of landraces of sorghum and millet from dry and humid regions. Ann. Bot. (Lond.) 57:835-846.

Cohen, S. 1998. A guide to the polyamines. Oxford University Press, New York, NY.

Dai, Y.-R., R. Kaur-Sawhney, and A.W. Galston. 1982. Promotion by gibberellic acid of polyamine biosynthesis in internodes of lightgrown dwarf peas. Plant Physiol. 69(1):103-106.

Davies, P.J. 1995. Plant hormones. Kluwer Academic Publishers, Dordrecht, The Netherlands.

Dhindsa, R.S., P.P. Dhindsa, and T.A. Thorpe. 1981. Leaf senescence: Correlation with increased levels of membrane permeability and lipid peroxidation, and decreased levels of SOD and CAT. J. Expt. Bot. 32:93-101.

Ervin, E. and J. Koski. 2001. Trinexapac-ethyl increases kentucky bluegrass leaf cell density and chlorophyll concentration. HortScience 36:787-789.

Gill, S.S. and N. Tuteja. 2010. Polyamines and abiotic stress tolerance in plants. Plant Signal. Behav. 5:26-33.

Gupta, S., V.P. Agarwal, and N.K. Gupta. 2012. Efficacy of putrescine and benzyladenine on photosynthesis and productivity in relation to drought tolerance in wheat (Triticum aestivum L.). Physiol. Mol. Biol. Plants 18(4):331-336.

Heath, R.L. and L. Packer. 1968. Photoperoxidation in isolated chloroplasts. Arch. Biochem. Biophys. 125:189-198.

Jimenez-Bremont, J.F., M. Marina, M.D. Guerrero-Gonzalez, F.R. Rossi, D. Sanchez-Rangel, M. Rodriguez-Kessler, O. Ruiz, and 
A. Garriz. 2014. Physiological and molecular implications of plant polyamine metabolism during biotic interactions. Front. Plant Sci. 5:1-14.

Kotakis, C., E. Theodoropoulou, K. Tassis, C. Oustamanolakis, N.E. Ioannidis, and K. Kotzabasis. 2014. Putrescine, a fast-acting switch for tolerance against osmotic stress. J. Plant Physiol. 171:48-51.

Krishnan, S. and E.B. Merewitz. 2014. Drought stress and trinexapacethyl modify phytohormone content within kentucky bluegrass leaves. J. Plant Growth Regulat. 34:1-12.

Krishnan, S. and E.B. Merewitz. 2015. Phytohormone responses and cell viability during salinity stress in two creeping bentgrass cultivars differing in salt tolerance. J. Amer. Soc. Hort. Sci. 140(4):346-355.

Kusano, T., K. Yamaguchi, T. Berberich, and Y. Takahashi. 2007. Advances in polyamine research in 2007. J. Plant Res. 120:345-350. Kyriakidis, D.A. 1983. Effect of plant growth hormones and polyamines on ornithine decarboxylase activity during the germination of barley seeds. Physiol Plant 57:499-504.

Liu, H., X. Li, J. Xiao, and S. Wang. 2012. A convenient method for simultaneous quantification of multiple phytohormones and metabolites: Application in study of rice-bacterium interaction. Plant Methods 8:2.

Lu, S., W. Su, H. Li, and Z. Guo. 2009. Abscisic acid improves drought tolerance of triploid bermudagrass and involves $\mathrm{H} 2 \mathrm{O} 2-$ and NO induced antioxidant enzyme activities. Plant Physiol. Biochem. 47:132-138.

Pottosin, I. and S. Shabala. 2014. Polyamines control of cation transport across plant membranes: Implications for ion homeostasis and abiotic stress signaling. Front. Plant Sci. 5:154.
Rachmilevitch, S., M. DaCosta, and B. Huang. 2006. Physiological and biochemical indicators for stress tolerance, p. 321-356. In: B. Huang (ed.). Plant-environment interactions. CRC Press, Boca Raton, FL.

Roychoudhury, A., S. Basu, and D.N. Sengupta. 2011. Amelioration of salinity stress by exogenously applied spermidine or spermine in three varieties of indica rice differing in their level of salt tolerance. J. Plant Physiol. 168:317-328.

Saleethong, P., J. Sanitchon, K. Kong-ngern, and P. Theerakulpisut. 2013. Effects of exogenous spermidine (Spd) on yield, yield related parameters and mineral composition of rice (Oryza sativa L. ssp. indica) grains under salt stress. Austral. J. Crop Sci. 7:1293-1301.

Shi, H., T. Ye, and Z. Chan. 2013. Comparative proteomic and physiological analyses reveal the protective effect of exogenous polyamines in the bermudagrass (Cynodon dactylon) response to salt and drought stresses. J. Proteome Res. 12:4951-4964.

Shiozaki, S., X. Zhuo, T. Ogata, and S. Horiuchi. 1998. Involvement of polyamines in gibberellin induced development of seedless grape berries. Plant Growth Regulat. 25:187-193.

Shukla, V., Y.M. Ma, and E.B. Merewitz. 2015. Creeping bentgrass responses to drought stress and polyamine application. J. Amer. Soc. Hort. Sci. 140:94-101.

Yamaguchi, S. 2008. Gibberellin metabolism and its regulation. Annu. Rev. Plant Biol. 59:225-251.

Yamaguchi, K., Y. Takahashi, T. Berberich, A. Imai, T. Takahashi, A.J. Michael, and T.A. Kusano. 2007. Protective role for the polyamine spermine against drought stress in Arabidopsis. Biochem. Biophys. Res. Commun. 352:486-490. 